\title{
LES ACTIONS DU SERVICE PÉDAGOGIQUE DU CENTRE D'ÉTUDES ALEXANDRINES
}

\author{
MARWA ABDEL GAWAD \\ marwa.abdelgawad@cea.com.eg \\ CENTRE D’ÉTUdES ALEXANDRINES (CEALEX, USR 3134 CNRS) \\ CHARLOTTE GLEIZE \\ ch.gleize@gmail.com \\ VSI, PACA, FranCE-VolontAires, CENTRE D'Études AleXANDrines \\ (CEALEX, USR 3134 CNRS) \\ http://dx.doi.org/10.25267/Riparia_sup.2018.11.16
}

Le Service pédagogique du Centre d'Études Alexandrines (CEAlex), Unité de Service et de Recherche 3134 du CNRS, a été créé en 2002, avec le soutien financier et technique du Conseil Régional Provence-Alpes-Côte d'Azur (PACA) et de l'association France Volontaires, en partenariat avec le Gouvernorat d'Alexandrie, dans le cadre de la coopération en Méditerranée.

Ses missions permettent aux Alexandrins, toujours plus nombreux à s'intéresser à leur patrimoine, de s'initier aux métiers de l'archéologie, de suivre les travaux des scientifiques et les avancées de la recherche, de visiter les sites et les monuments de leur ville et de s'ouvrir sur les civilisations méditerranéennes.

Pour ce faire, le Service pédagogique du CEAlex opère autour de trois axes principaux :

- l'animation d'activités périscolaires ;

- la programmation d'événements culturels ;

- l'organisation de formations.

Grâce à ces actions, les Alexandrins découvrent ou redécouvrent l'histoire de leur ville et peuvent s'approprier la richesse de son patrimoine. Un patrimoine historique, culturel et également naturel.

\section{L'animation d'activités périscolaires}

L'animation des activités périscolaires constitue le fil rouge des actions du Service pédagogique du CEAlex tout au long de l'année. Les établissements scolaires, privés et publics, ainsi que certaines institutions culturelles, au premier rang desquelles la Bibliotheca Alexandrina, font appel au Service pédagogique pour animer des visites de sites, pour fournir des mallettes pédagogiques et pour organiser des ateliers (fig. 1). Chaque année, ce n'est pas moins de 30 établissements qui sollicitent le Service pédagogique pour venir sensibiliser près de 6500 jeunes alexandrins au patrimoine de leur ville. 


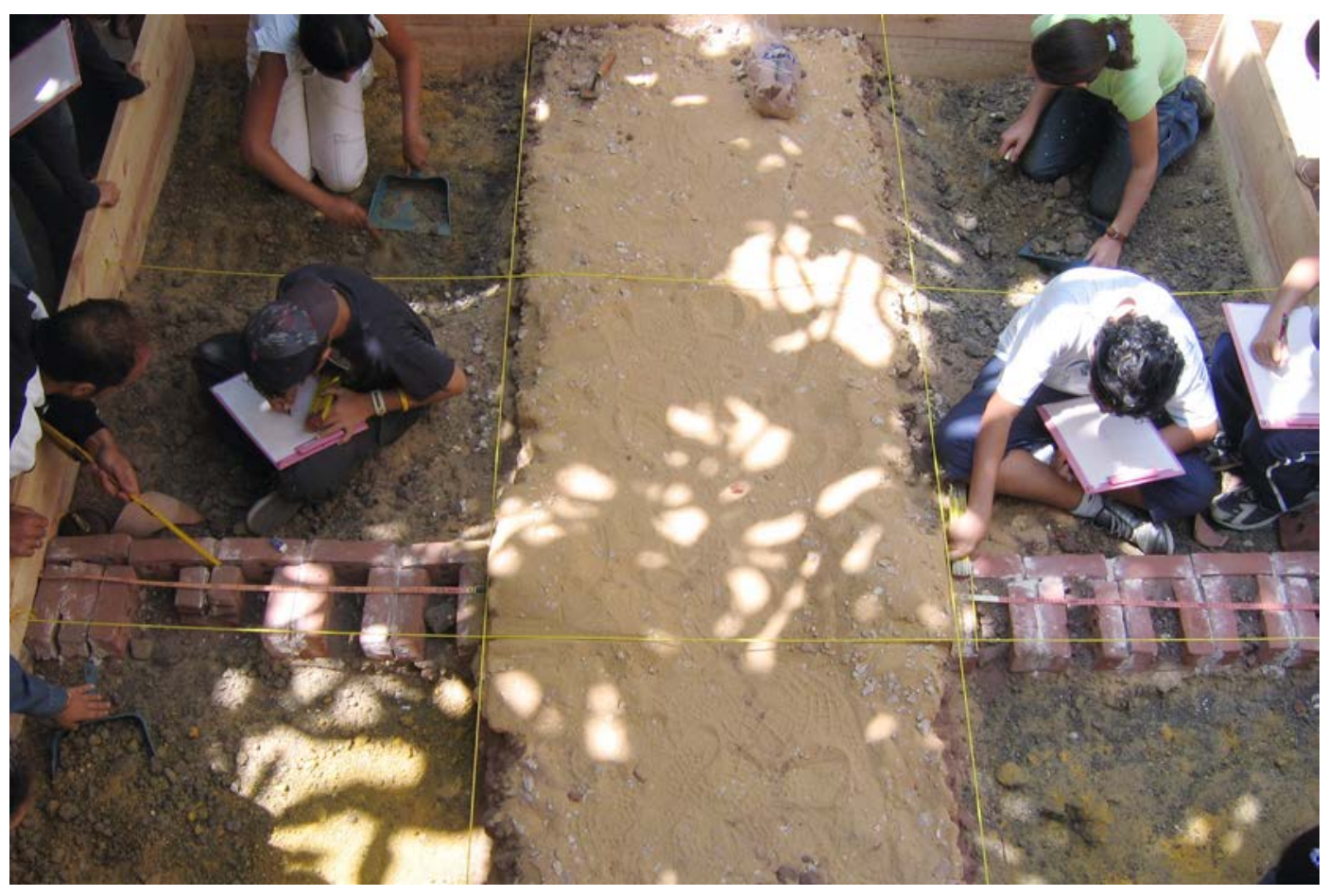

Fig. 1. La vraie-fausse fouille, École Champollion, Alexandrie (Archives CEAlex/CNRS).

Expliquer l'héritage historique de la ville d'Alexandrie doit passer par la présentation des sites que les archéologues ont mis au jour. Les vestiges encore visibles dans la ville sont aujourd'hui peu nombreux mais suffisamment éloquents pour enrichir les enseignements d'histoire reçus en classe. Ils illustrent plusieurs périodes de l'histoire, de l'Antiquité à l'époque moderne, et permettent ainsi de proposer aux enseignants plusieurs visites thématiques couvrant différentes périodes historiques. De plus, en complément d'un approfondissement apporté aux cours d'histoire, ces visites sont l'occasion de présenter les disciplines de la recherche et la pratique de l'archéologie, souvent méconnues du jeune public. Elles permettent en outre de sensibiliser les jeunes visiteurs à la protection du patrimoine et à l'importance de sa conservation au fil du temps. En partenariat avec le Ministère des Antiquités et le Gouvernorat d'Alexandrie, le Service pédagogique propose la visite de six sites emblématiques : le Sérapeum, les Catacombes de Kôm el-Chougafa, le dépôt archéologique de Shallalat, la citadelle de Qaitbay, le Musée National d'Alexandrie et le Théâtre romain auquel est accolé le Musée en plein air exposant certaines œuvres statuaires colossales issues du phare d'Alexandrie et des monuments pharaoniques, découvertes et remontées des eaux par les archéologues du CEAlex. Les visites, assurées par un membre du Service pédagogique, sont accompagnées de fascicules d'informations et d'animations, distribués aux élèves, pour prolonger l'enseignement reçu sur le site.

Les mallettes pédagogiques constituent un autre moyen pour sensibiliser le jeune public au patrimoine et en élargir les champs de définition (fig. 2). Le Service pédagogique a conçu plusieurs mallettes associées à diverses thématiques pour aborder des questions en partie orientées vers le patrimoine naturel. Les membres du Service pédagogique réunissent chaque année les enseignants et responsables de cycle afin de les former à l'animation de ces mallettes. Ainsi, chaque établissement peut emprunter une mallette pour un temps défini et diriger les activités de la mallette selon le programme d'étude des professeurs. Les membres du Service pédagogique se 
proposent également de venir dans les écoles, collèges et lycées, dont les enseignants n'auraient pas pu suivre la formation. À titre d'exemple, citons deux mallettes pédagogiques particulièrement appréciées du corps enseignant et des élèves.

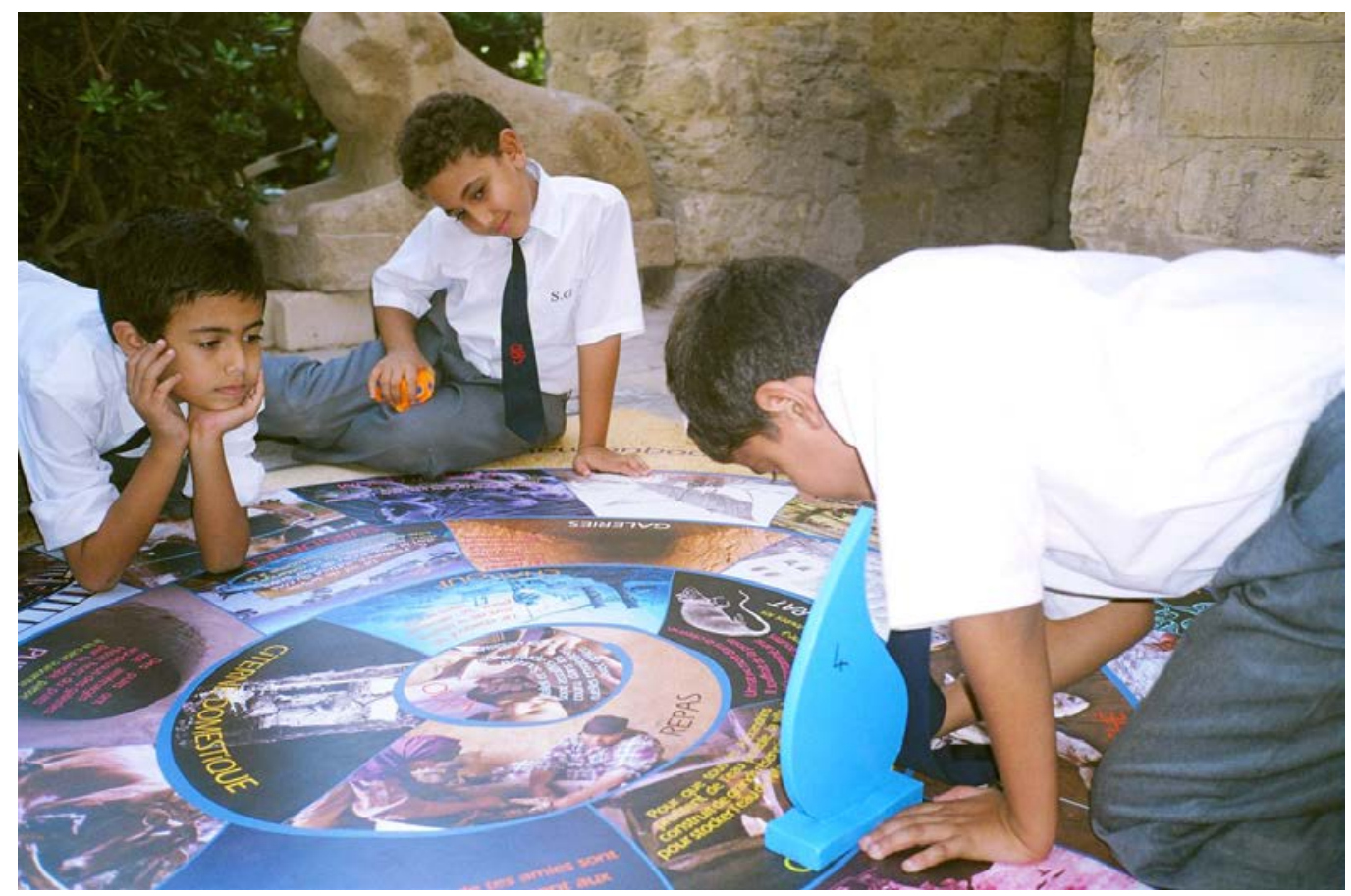

Fig. 2. Le jeu de l'eau dans le jardin du musée gréco-romain d'Alexandrie (Archives CEAlex/CNRS).

Créée en partenariat avec l'association Les petits débrouillards de Marseille, la mallette Archéeau regroupe trois activités tournées vers la discipline de l'archéologie sous-marine et la problématique de l'eau à Alexandrie. Grâce aux trois activités de cette mallette proposant expériences scientifiques et ateliers de rédaction, les participants peuvent comprendre les propriétés de l'eau, sa gestion dans une ville antique et l'importance de son entretien à l'heure actuelle.

Une autre mallette que propose le Service pédagogique, Le tapis volant, a été conçue en 2013 par l'association partenaire Bokra Sawa, établie à Marseille. Cette mallette propose quatre activités qui présentent l'histoire du Canal de Suez, l'impact économique et sociale de son ouverture à l'échelle mondiale et sensibilise aux questions de biodiversité marine et de pollution en milieux terrestre et sous-marin.

À côté de ces animations ponctuelles, le Service pédagogique du CEAlex propose également aux établissements scolaires et institutions culturelles des activités qui se déroulent sur plusieurs séances afin d'approfondir de manière ludique un sujet en lien avec le patrimoine d'Alexandrie. Ces ateliers sont l'occasion d'aborder des thèmes variés qui élargissent encore un peu plus la notion de patrimoine à l'histoire de l'art, à la littérature, mais aussi aux notions de conservation et de transmission de ce patrimoine. La création de ces ateliers s'est faite avec le concours des chercheurs du CEAlex qui ont apporté leurs savoirs respectifs sur les différents sujets abordés. Le Service des archives qui coordonne le programme Presse Francophone d'Égypte a notamment collaboré à la création de trois ateliers sur le surréalisme, le journalisme (fig. 3) et les archives à Alexandrie à l'époque contemporaine. Ces ateliers permettent 
de présenter ces disciplines, leurs spécificités à Alexandrie, et proposent aux participants de devenir eux-mêmes acteurs du patrimoine par la conception de supports en lien avec le thème abordé.

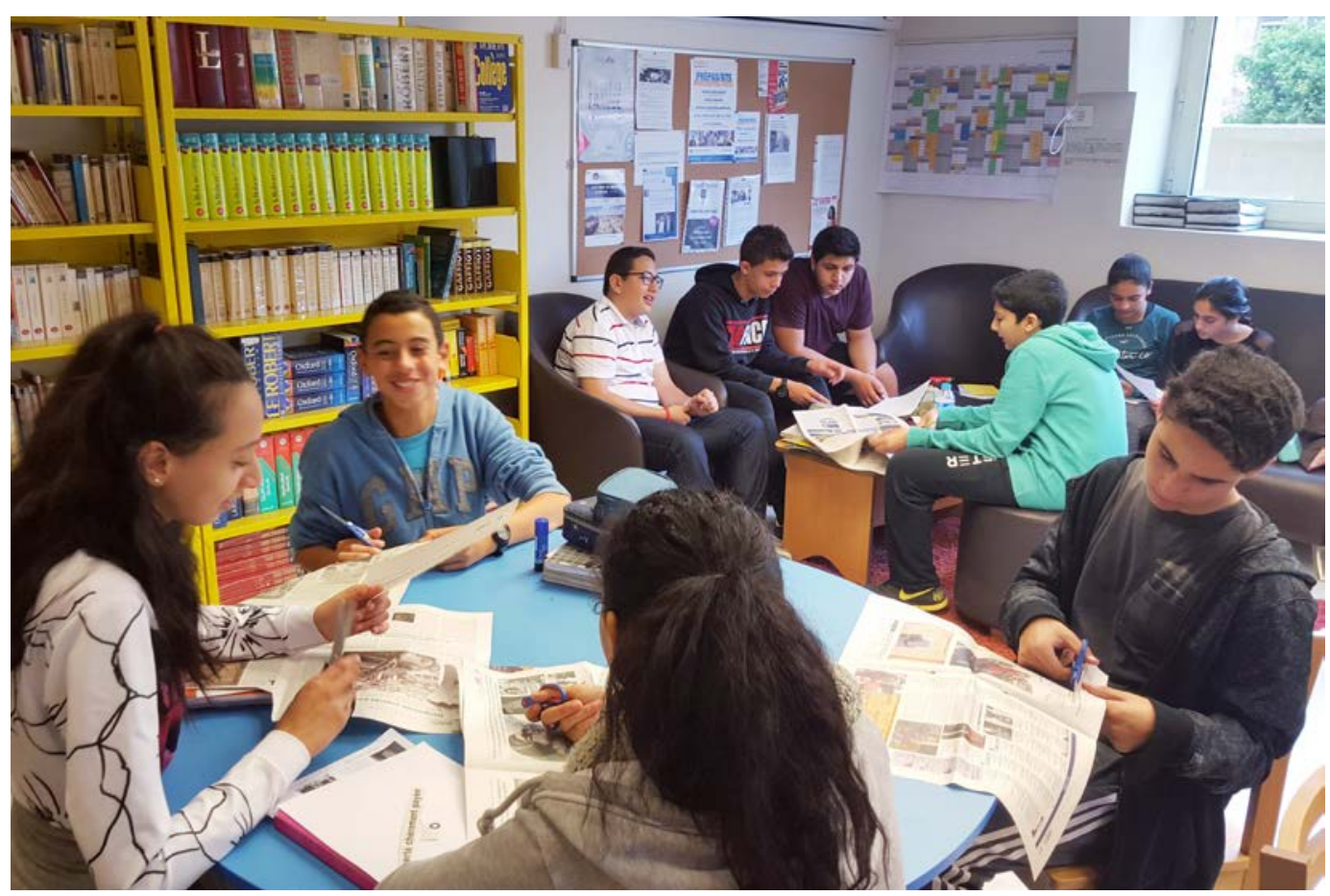

Fig. 3. Atelier « journalisme », Lycée français d'Alexandrie (Archives CEAlex/CNRS).

Ces activités périscolaires permettent d'introduire auprès du jeune public la notion même de patrimoine, d'en présenter une large étendue, spécifique à la ville d'Alexandrie, et de sensibiliser les participants à sa conservation, grâce à de nombreuses animations tenues tout au long de l'année. De nouveaux programmes d'études, actuellement en préparation, seront proposés aux enseignants afin de couvrir l'étendue du champ patrimonial d'Alexandrie depuis l'Antiquité jusqu'à l'époque contemporaine autour de thématiques originales en lien avec les travaux de recherches du CEAlex.

\section{La programmation d'événements culturels}

La programmation d'événements culturels constitue le deuxième volet des activités du Service pédagogique du CEAlex. Organisés annuellement et à destination du grand public, ces moments de rassemblement et de festivités sont voués à la promotion du patrimoine alexandrin et méditerranéen. En collaboration avec de nombreuses institutions égyptiennes, françaises et étrangères, issues des milieux culturels, scientifiques et diplomatiques, le Service pédagogique assure la programmation et la coordination de ces manifestations qui, chaque année, rassemblent pas moins de 35 partenaires et ont été fréquentées par 5000 visiteurs en 2016.

Inspirées des Journées Européennes du Patrimoine, les Journées du patrimoine alexandrin connaissent un succès croissant depuis leur première édition en 2010. Cet événement qui se déroule annuellement en novembre propose le temps d'une semaine de faire partager à un large public les richesses patrimoniales et culturelles de 
la ville d'Alexandrie, à travers son histoire, son architecture, son urbanisme et, bien sûr, ses acteurs. Chaque année, des partenaires présents depuis la création de l'événement, mais aussi de nouvelles institutions répondent à l'appel et proposent des manifestations inédites autour d'un thème défini en amont par le CEAlex. Conférences, visites guidées de sites et monuments, spectacles d'arts de la scène et d'arts visuels, expositions (fig. 4), ateliers et bien d'autres activités, sont organisés dans les différents quartiers de la ville où le public prend place et participe ainsi au rayonnement de son patrimoine.

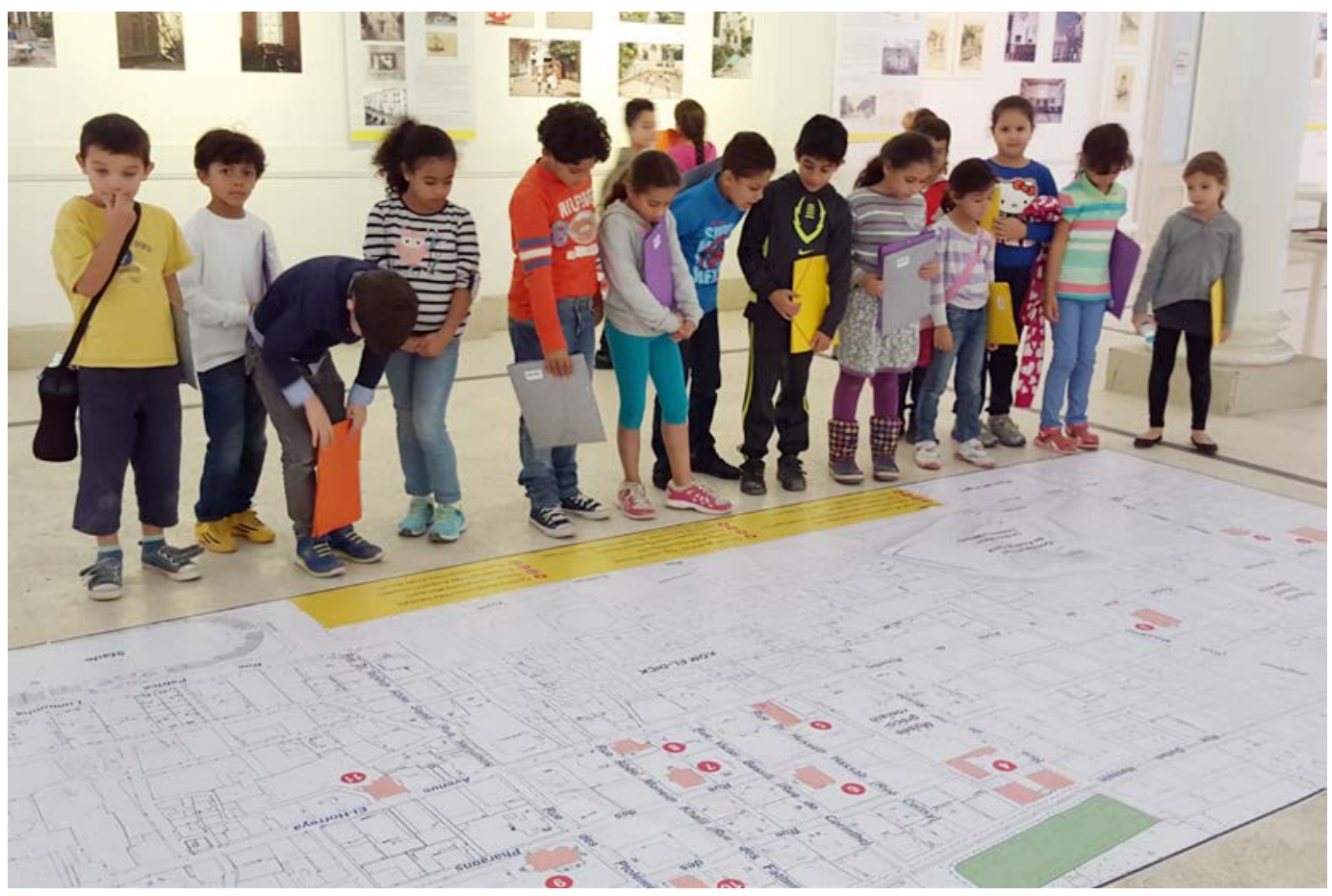

Fig. 4. Animation autour de l'exposition "Les écoles dans les palais» créée par le CEAlex dans le cadre des journées du patrimoine alexandrin 2016, Institut français d'Égypte à Alexandrie. Archives

CEAlex/CNRS.

Autre temps fort du calendrier, voué à perdurer pour les années à venir : les Journées AA, soit les Journées de l'Archéologie à Alexandrie, dont la première édition a eu lieu en 2016. Issue d'une collaboration avec l'INRAP dans la médiation des Journées Nationales de l'Archéologie, la création de ce nouvel événement s'est calquée sur les mêmes dates que son homologue français, les 17,18 et 19 juin. Ces trois jours d'activités ont permis de présenter les métiers de l'archéologie et les grandes découvertes qui ont marqué l'histoire de la ville et de l'Égypte à travers trois périodes: l'époque pharaonique, l'époque hellénistique et l'époque romaine. Pour cette première édition, le choix a été fait de collaborer principalement avec des institutions en lien avec le jeune public; il est à envisager un élargissement des partenaires et du public pour les prochaines éditions, pour que le plus grand nombre puisse accéder aux savoirs sur l'archéologie terrestre et sous-marine d'Alexandrie. 
Le Service pédagogique participe également en tant que partenaire à la Fête de la Science, organisée depuis 2007 par la Bibliotheca Alexandrina et le Centre des Sciences du Planetarium. Chaque année, durant les deux jours de festivités, le Service pédagogique présente une partie du patrimoine d'Alexandrie en lien avec le thème annoncé par les organisateurs. En 2016, la thématique de la santé a offert l'opportunité au Service pédagogique de sensibiliser les visiteurs à la transmission des savoirs des sciences médicales grecques dans le bassin méditerranéen, de souligner l'importance de l'école de médecine d'Alexandrie à l'époque gréco-romaine et de présenter l'enrichissement des connaissances médicales grâce aux sciences arabes à l'époque médiévale. Partage des connaissances et influences des différentes civilisations sur le temps long ont permis de présenter le patrimoine alexandrin dans son contexte méditerranéen et de promouvoir ses héritages interculturels.

Depuis sa création, le Service pédagogique a également mené de nombreux projets en partenariat avec le Conseil Régional PACA, animés dans les villes de Marseille et d'Arles, en collaboration avec plusieurs institutions locales. Ces initiatives permettent d'ancrer Alexandrie dans son cadre méditerranéen et de présenter le patrimoine commun que peuvent partager ces différentes villes depuis leur création. Le dernier projet en date, $L a$ ville entre réel et imaginaire, conçu et réalisé en partenariat avec l'association Bokra Sawa, s'est déroulé en deux phases, l'une à Alexandrie à l'automne 2015 et l'autre à Marseille au printemps 2016. Faisant intervenir des professionnels de l'architecture et des arts plastiques, ce projet auquel ont participé de jeunes citoyens des deux villes a eu pour but la sensibilisation au patrimoine bâti et à l'urbanisme d'Alexandrie et de Marseille, au rythme de visites guidées de différents quartiers, d'ateliers de dessins et d'expositions temporaires présentant les travaux des participants.

Ces grands événements permettent chaque année de rassembler une grande partie des Alexandrins et de les rendre acteurs du patrimoine de leur ville. L'engouement du public pour ces festivités encourage le Service pédagogique à pérenniser ces manifestations et les faire résonner au-delà des frontières de la ville.

\section{L'organisation de formations}

Fort de son expérience, le Service pédagogique du CEAlex propose des sessions de formations auprès des établissements scolaires et universitaires qui souhaitent participer aux activités périscolaires et approfondir leur savoir-faire dans la création et la coordination de projets pédagogiques. Des séances sont également organisées auprès des inspecteurs du Ministère des Antiquités pour la médiation culturelle auprès du jeune public.

Chaque année, en amont de la rentrée des classes, le Service pédagogique convie les enseignants et responsables de cycles des établissements scolaires, publics et privés, afin de leur présenter les activités périscolaires proposées pour l'année à venir et de proposer des formations à ceux qui souhaitent animer les mallettes pédagogiques et certains ateliers auprès de leurs élèves. En outre, le Service pédagogique collabore avec l'Université d'Alexandrie et l'Université francophone Léopold Senghor et intervient dans la formation des étudiants se destinant aux métiers de l'animation et de la médiation auprès du jeune public.

Chaque année également, dans le cadre d'un partenariat avec le Ministère des Antiquités et en collaboration avec les nombreuses institutions patrimoniales d'Alexandrie, le Service pédagogique propose une formation à destination des inspecteurs de sites et du personnel des musées en charge de la médiation. Ces formations comportent une partie théorique : rendus d'expériences et études de cas 
rythment les premières séances. S'en suit une partie pratique durant laquelle les inspecteurs doivent présenter un site, concevoir des parcours thématiques et proposer des activités spécifiques.

Ces séances de formations constituent le moment privilégié pour tous les professionnels et futurs professionnels des métiers de l'animation, de la médiation et de l'enseignement, pour échanger connaissances et compétences et créer un réseau de spécialistes des métiers du patrimoine dans la ville d'Alexandrie.

Grâce à l'ensemble de ses activités, le Service pédagogique du CEAlex se présente aujourd'hui comme un interlocuteur majeur pour répondre aux questions autour de la présentation, de la sensibilisation et du rayonnement du patrimoine alexandrin, au sein même de la ville et au-delà de ses frontières. Sensibiliser la population sur son patrimoine passe d'abord par l'éducation et c'est aux jeunes générations qu'il convient en premier lieu de s'adresser pour que l'héritage qu'ils recevront perdure encore pour longtemps. 